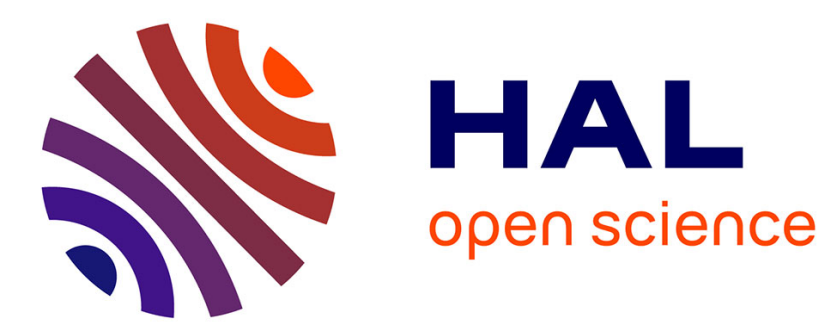

\title{
Creases and cracks in finite elasticity
}

Martine Ben Amar

\section{To cite this version:}

Martine Ben Amar. Creases and cracks in finite elasticity. Mechanics Research Communications, 2018, 93 (Octobre 2018), pp.23-29. hal-01969792

\section{HAL Id: hal-01969792 https: / hal.sorbonne-universite.fr/hal-01969792}

Submitted on 4 Jan 2019

HAL is a multi-disciplinary open access archive for the deposit and dissemination of scientific research documents, whether they are published or not. The documents may come from teaching and research institutions in France or abroad, or from public or private research centers.
L'archive ouverte pluridisciplinaire HAL, est destinée au dépôt et à la diffusion de documents scientifiques de niveau recherche, publiés ou non, émanant des établissements d'enseignement et de recherche français ou étrangers, des laboratoires publics ou privés. 


\title{
Creases and cracks in finite elasticity
}

\author{
Martine Ben Amar ${ }^{1}$ \\ Laboratoire de Physique Statistique, Départemant de Physique de l'ENS,PSL Research University, Sorbonne Université, UPMC Univ. Paris 06, CNRS, 75005 \\ Paris \\ Institut Universitaire de Cancérologie, Faculté de médecine, Université Pierre et Marie Curie-Paris 6, 91 Bd de l'Hôpital, 75013 Paris, France
}

\begin{abstract}
This paper contains a model of singularities occurring in soft tissues due to resorption of matter or drying. An asymptotic treatment, consistent with the fully nonlinear equilibrium theory near the tip of a crack in a slab is considered in plane strain, plane stress and poro-elastic modeling. The crack faces are free of stresses but capillary effects or surface elasticity are also included. The tensile loading applied at infinity is due to the resorption of matter or to fluid flow caused by drying. The asymptotic analysis is completed by the technique of the $J$-integral and the stress intensity factor can be evaluated in the three cases.
\end{abstract}

Keywords: Volumetric Growth and Drying; Spatial Singularity; Path-Independent Integrals; Nonlinear finite Elasticity;Fracture.

\section{1. introduction}

Creases have always fascinated theorists. Observed in material sciences, they concern foldings [1], D-cones (developable cones) [2], origami [3] and also delamination [4]. The main idea is that a system under loading focuses its elastic energy in a small area instead of spreading it all over the sample. The simplest example is furnished by the Foppl Von Karman equation [5] where the juxtaposition of a bending energy in $h^{3}$ (where $\mathrm{h}$ is the thickness of the sample) is discarded in comparison with 10 the stretching energy in $h$, forcing the sample to adopt a de- 40 velopable surface [6]. Since these surfaces do not match the boundaries most of the time, it results a crumpling, combination of conic points and folds. In swelling experiments, creases have been experimentally observed going back to Tanaka's series of papers [7, 8], more recently in [9, 10, 11]. Occurring in growth processes, such creases are also revealed in numerical studies [12, 13]. Since their existence is not fully explained in volumetric growth, it remains a hot topic for theorists.

On the contrary, a more well established subject in finite elasticity concerns cracks. Cracks are commonly observed when 50 a gel or an emulsion loose water as clay soils in hot weather (see Fig.(1)). This figure exhibits a network of fractures rather geometrical and is an illustration that drying induces tensile stresses in thin layers able to nucleate fractures. There is no loading here as in conventional fracture set-ups and the stresses originate from the loss of water, then corresponding to the poroelastic model. The geometry of such network has been mostly studied numerically with an hydraulic perspective [14] where fluid flows are important. A more physical interpretation of the

Email address: benamar@lps.ens.fr (Martine Ben Amar)
30 fracture branching can also be found in [15, 16], based on successive crack formations where a primary set of fractures is followed by secondary cracks joining the primary network at right angles. Such angular distribution allows the maximal relaxation of stresses by fracturing in a tensorial field. Clearly, the geometrical aspect of these fracture networks is an interesting topic in itself but the process requires a treatment in finite elasticity, more precisely poro-elasticity which has not been achieved yet, to the best of our knowledge. Nevertheless, even if such pattern is out of the scope of this present work, the poro-elastic model is presented in the context of fracture here. Fracture is an important classical domain of applied mechanics and material sciences which remains very active nowadays. As an example, recently the technique of laser nano-fracture in living embryos has motivated new modeling of fracture shape in orthotropic elasticity with orthogonal tensile loadings [17]. Exact solutions for the repartition of stresses have been found in applied mathematics with the techniques of holomorphic functional analysis in ideal loading conditions defined by elementary modes (mode I in tension, II and III for shear modes). But even when it was impossible to find exact solutions, physical features can been explained without solving completely the stresses. It is the case for the time scale for break-down [18], the dynamics of the cracks under loading [19], the path of fracture propagation which may exhibit or not instabilities or branching [20]. Indeed, even in linear elasticity, the fact that only few exact solutions have been discovered [21] for simplified geometries, explains why theoretical models have been elaborated without solving all the details of the elastic stresses in the sample. In particular, many physical results can be predicted from the so called stress intensity factor [22], derived from the stress at the tip. Since a local analysis allows to reach this quantity in linear elasticity, it is tempting to adopt the same strategy for finite elasticity 
and cracks that we will assume here generated by desorption or drying. In this case, it is possible to compare mode I of fracture mechanics (mode of fracture only in tension) with the state of tension generated by resorption of matter. We will show that, even in $2 \mathrm{D}$, it is not always easy or possible to establish such a correct local treatment near the tip of a crack according to the nonlinearities. If, for the Neo-Hookean model, the analysis is 70 relatively easy in plane strain elasticity, it is more challenging in plane stress.

Our aim is then to demonstrate that a local analysis remains possible, then showing evidence of the existence of a crack under resorption of matter or drying. We will illustrate the method on three different cases: two cases on volumetric resorption in Neo Hookean models in plane strain and plane stress in the strip geometry or for an infinite periodic array of cracks. We also consider the poro-elastic model which is a better representation of gel drying. To complete the local analysis, the path80 independent $J$-integral [23] is used which allows to connect the crack opening at the tip as a function of the drying conditions imposed at long distances. Extensions [25, 26] or variants of this integral [27] to more complex problems of finite elastostatic have been achieved by G. Maugin and collaborators.

Section 2 presents the three models with the associated EulerLagrange equations and boundary conditions including capillarity from variation of the elastic energy. Section 3 is devoted to the asymptotic analysis performed around the tip of the crack in the three models. Section 4 gives the scaling at the tip or the 90 stress intensity factor by the technique of the J-integral. Finally Section 5 contains conclusions and perspectives about this analysis.

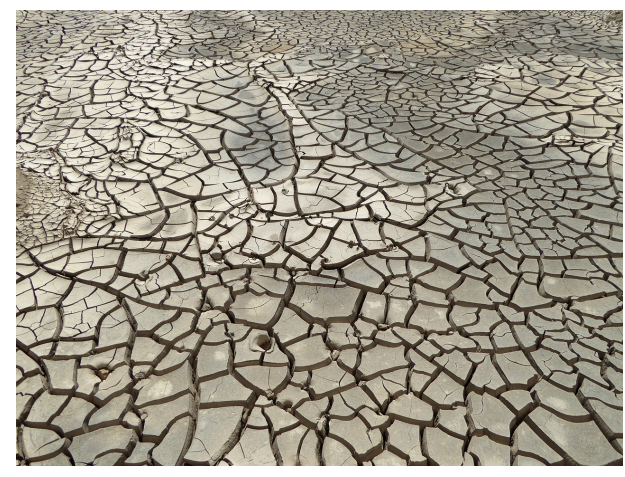

Figure 1: Dry clay soil. Observe the geometry of the network of fractures. Pixabay image

\section{The Models}

We first consider the volumetric growth of a sample, infinite in the $Y$ direction: $-\infty<Y<\infty$, also infinite in the positive $X$ direction. The upper horizontal border is located at $X=-L$, in the reference configuration. The origin of the axis is fixed at the crack tip: $(X=0, Y=0)$. We restrict on the Neo-Hookean model but the results can be extended to more complex nonlinear elasticity models, allowing much more possibilities by the multiplication of degrees of freedom. The $Z$ direction is perpendicular to the plane considered (see Fig.(2.1)). The growth tensor $\mathbf{G}$ is taken diagonal in each direction $(X, Y, Z)$ for volumetric changes, in plane strain or stress (but remains isotropic for the gel modeling) giving $\mathbf{G}=\operatorname{Diag}\left(g_{1}, g_{2}, g_{3}\right)$.

\subsection{Volumetric changes in plane strain elasticity}

First, the deformation gradient tensors, the geometric tensor $\mathbf{F}$ and elastic tensor $\mathbf{F}_{\mathbf{e}}$, satisfy the relation $\mathbf{F}_{\mathbf{e}}=\mathbf{F} \mathbf{G}^{-1}$ in the volumetric growth hypothesis [28, 29]. It reads:

$$
\mathbf{F}=\left[\begin{array}{ccc}
x_{\tilde{X}} & x_{\tilde{Y}} & 0 \\
y_{\tilde{X}} & y_{\tilde{Y}} & 0 \\
0 & 0 & z_{\tilde{Z}}
\end{array}\right] \quad \mathbf{F}_{\mathbf{e}}=\left[\begin{array}{ccc}
x_{\tilde{X}} / g_{1} & x_{\tilde{Y}} / g_{2} & 0 \\
y_{\tilde{X}} / g_{1} & y_{\tilde{Y}} / g_{2} & 0 \\
0 & 0 & z_{\tilde{Z}} / g_{3}
\end{array}\right],
$$

where $x_{\tilde{X}}=\partial x / \partial \tilde{X}$ and equivalent relationships for the other coordinates. The material is assumed incompressible, which implies Det $\left(\mathbf{F}_{\mathbf{e}}\right)=1$ and the incompressibility constraints implies that the Jacobian $\tilde{J}_{2 d}$

$$
\tilde{J}_{2 d}=\lambda_{3}\left(\frac{\partial x}{\partial \tilde{X}} \frac{\partial y}{\partial \tilde{Y}}-\frac{\partial x}{\partial \tilde{Y}} \frac{\partial y}{\partial \tilde{X}}\right)=g_{1} g_{2}
$$

where $\lambda_{3}$ is the stretch, assumed constant in the third direction to satisfy the condition of plane strain elasticity. During growth or shrinking, the elastic energy is then:

$$
\mathcal{E}=g_{1} g_{2} g_{3} \frac{\mu}{2} \int_{\tilde{V}}\left(\operatorname{Trace}\left[\mathbf{F}_{e} \mathbf{F}_{e}^{T}\right]-3-2 \tilde{p}\left(\tilde{J}_{2 D}-g_{1} g_{2} / \lambda_{3}\right)\right) d \tilde{V}
$$

$\tilde{V}$ being the volume in the reference configuration before growth, $\mu$ the shear modulus and $\tilde{p}$ is the Lagrange parameter which allows to impose the constraint Eq.(2). At the boundary of the sample and on the crack lips, surface effects such as surface tension $T$ and surface elastic energy $\mathcal{E}_{S}$ can be treated. In particular for gels, the surface elastic energy has shown importance, see recent works [30, 31]

$$
T=\int_{\bar{s}} \gamma(\vec{n}) d \bar{s} ; \quad \mathcal{E}_{S}=g_{3} g_{i} \frac{\mu_{s}}{2} \int_{S}\left(\frac{\partial x_{i}}{\partial \tilde{X}_{i}}\right)^{2} d \tilde{Z} d \tilde{X}_{i}
$$

where $\bar{s}$ is the surface of the free boundary in the current configuration and the index $i$ corresponds to the surface coordinate. After rescaling the coordinates $(\tilde{X}, \tilde{Y})$ by $X=g_{1} \tilde{X}$, $Y=g_{2} \tilde{Y}, Z=g_{3} \tilde{Z}$ and transforming the Lagrange parameter into $p=g_{1} g_{2} \tilde{p}$, it is possible to eliminate the growth eigenvalues from the elastic energy per unit length in the third direction which gives:

$\mathcal{E}=\frac{\mu}{2} \int_{S}\left\{x_{X}^{2}+x_{Y}^{2}+y_{X}^{2}+y_{Y}^{2}+\lambda_{3}^{2}-3-2 p\left(J_{2 D}-1 / \lambda_{3}\right)\right\} d X d Y$

where $J_{2 D}=x_{X} y_{Y}-x_{Y} y_{X}$. To simplify in the following, we restrict on $\lambda_{3}=1$. The Euler-Lagrange equation can be easily found (see also [32]):

$$
\left\{\begin{array}{c}
\Delta x=p_{X} y_{Y}-p_{Y} y_{X} \\
\Delta y=p_{Y} x_{X}-p_{X} x_{Y}
\end{array}\right\}
$$

where $\Delta$ is the two-dimensional Laplacian operator. Boundary conditions depend on the free surface under consideration. Focussing on the upper boundary where $X=-L$, we have:

$$
\left\{\begin{array}{lll}
s_{11}=x_{X}-p y_{Y}=d_{0} \frac{d}{d Y} \frac{x_{Y}}{\sqrt{x_{Y}^{2}+y_{Y}^{2}}} & \text { for } & X=-L \\
s_{21}=y_{X}+p x_{Y}=d_{0} \frac{d}{d Y} \frac{y_{X}}{\sqrt{x_{Y}^{2}+y_{Y}^{2}}} & \text { for } & X=-L
\end{array}\right\}
$$


where $d_{0}=\gamma / \mu$. For the boundary conditions along the lips of ${ }_{110}$ the creases $(\mathrm{Y}=0)$, it comes:

$$
\left\{\begin{array}{lll}
s_{22}=y_{Y}-p x_{X}=d_{0} \frac{d}{d X} \frac{y_{X}}{\sqrt{x_{X}^{2}+y_{X}^{2}}} & \text { for } & Y=0 \\
s_{12}=x_{Y}+p y_{X}=d_{0} \frac{d}{d X} \frac{x_{X}}{\sqrt{x_{X}^{2}+y_{X}^{2}}} & \text { for } & Y=0
\end{array}\right\}
$$

where $\mathbf{s}$ is the nominal stress. Let us consider now how these boundary conditions are modified if we incorporate a surface energy term [30, 31]. We restrict on the crack face $Y=0$. Then, coming back to the variational analysis, it is easy to show that only the normal stress is modified resulting in:

$$
s_{22}=d_{0} \frac{d}{d X} \frac{y_{X}}{\sqrt{x_{X}^{2}+y_{X}^{2}}}+d_{\mu} \frac{d}{d X}\left(x_{X}-p_{s}\right)
$$

where $p_{s}$ is the equivalent Lagrange parameter for the surface and $d_{\mu}=\mu_{s} / \mu$.

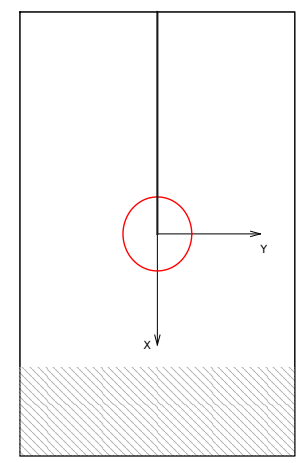

Figure 2: Crack representation in a strip of length $\Lambda$ in the reference configuration. The axis are defined at the tip. The sample is infinite in the positive $X$ direction. When the sample is infinite in the $Y$ direction, periodicity is postponed.

\subsection{Drying processes represented by the poro-elastic model}

Gels contain water and the drying cannot be described correctly by the Kröner-Lee decomposition. Indeed, during drying, water is ejected from the gel and we are confronted to a solid mixture of polymers where solvent molecules can move freely between a reservoir and the sample. In this case, the potential energy to minimize is indeed the Grand Potential $\hat{W}$ which is constructed via a Legendre transformation of the Helmholtz ${ }^{130}$ free energy density of the mixture $\mathcal{W}=\mathcal{W}(\mathbf{F}, \mathbf{C})$ where $\mathbf{F}$ is the deformation gradient and $C$ the solvent concentration. Incompressibility of all constituents gives Det $\mathbf{F}=\mathbf{1}+v \mathbf{C}, v C$ being the volume of the solvent divided by the volume of the dry network. Eliminating $C$, we finally derive (see also [10, 11]):

$$
\hat{\mathcal{W}}=\mathcal{W}\left(\mathbf{F},\left(\frac{\operatorname{Det}(\mathbf{F})-1}{v}\right)-\Pi(\operatorname{Det}(\mathbf{F})-1)\right.
$$

$\Pi$ is the dimensionless chemical potential scaled with $\mu$ and $v$. Notice that the last term in Eq. 10 is in fact a surface term. For $\mathcal{W}$, we adopt the generalization of the Neo-Hookean model [11] and the strain-energy density is then:

$$
\mathcal{W}=\frac{\mu}{2}\left(x_{X}^{2}+x_{Y}^{2}+y_{X}^{2}+y_{Y}^{2}-2-2 \log \operatorname{Det}(\mathbf{F})\right)
$$

The Euler-Lagrange equations and the boundary conditions, Eqs. (7.8), are the same as Eq. (6) providing the pressure is replaced by $1 / \operatorname{Det}(\mathbf{F})$. But, the stresses given in Eqs. (7/8) concerning free borders do not vanish, they must balance the "chemical stress" and we have: $\mathbf{s}^{T} \vec{N}=\Pi\left(\operatorname{Det}(\mathbf{F}) \mathbf{F}^{-\mathbf{T}}\right) \vec{N}$ which at the crack surface, gives surface terms like : $\delta s_{22}=$ $(\Pi)(\partial x / \partial X)$ and $\delta s_{12}=(\Pi)(-\partial y / \partial X)$ to eventually add to capillarity and surface elastic stresses. Let us now consider the last model, supposed to be more adapted to thin membrane.

\subsection{Volumetric resorption in plane stress elasticity}

As suggested by the name itself of this modeling, plane stress elasticity treats deformations where the stress components only depend on the coordinates $(\mathrm{X}, \mathrm{Y})$. It means that for the deformation gradient tensor Eq. (1) remains correct, but the stretch in the vertical direction is a function of $(X, Y)$. Such modeling is proposed for thin plates of thickness $H$ in finite elasticity. The incompressibility constraint becomes:

$$
J_{3 d}=\lambda_{3}(X, Y)\left(\frac{\partial x}{\partial X} \frac{\partial y}{\partial Y}-\frac{\partial x}{\partial Y} \frac{\partial y}{\partial X}\right)=1
$$

where $\lambda_{3}$ is a function on $X$ and $Y$ only to satisfy the definition of plane stress modeling. The elastic energy becomes:

$$
\mathcal{E}_{3 D}=g_{3} H \frac{\mu}{2} \int_{S}\left\{\mathcal{D}_{2 D}+\lambda_{3}^{2}-3-2 p_{3 D}\left(\lambda_{3} J_{2 D}-1\right)\right\} d X d Y
$$

where we have introduced the elastic energy density: $\mathcal{D}_{2 D}=$ $x_{X}^{2}+x_{Y}^{2}+y_{X}^{2}+y_{Y}^{2}-2$. Elementary variation of $\mathcal{E}_{3 D}$ with respect to $\lambda_{3}$ gives: $p_{3 D}=\lambda_{3} / J_{2 D}=\lambda_{3}^{2}$. So the plane stress EulerLagrange equations can be derived from Eqs. 678 8) by replacing $p$ by $\lambda_{3}^{3}$ everywhere, taking into account Eq. 12. As seen later on, such a modification, plane strain versus plane stress, introduces a strong nonlinearity making more difficult the local analysis.

\section{Local analysis around a pre-existing crack}

In this section, a local asymptotic of the deformations and stresses in the vicinity of the pre-existing crack tip is performed. We focus on mode I of elasticity, it means that the crack is under tension. The fact that a regular analysis can be achieved is a proof that these cracks may exist in the framework chosen for the nonlinear elastic models. As mentioned in [33] and also in [34] for cracks in compressible materials, it is absolutely not always the case.

\subsection{Local treatment for the volumetric growth model}

In the close vicinity of the singularity, a local frame is defined in polar coordinates, transforming the $(X, Y)$ axis into $(R, \Theta)$. Remember that $X$ and $Y$ are rescaled coordinates via the growth eigenvalues, so also $R$ and $\Theta . R=0$ is chosen at the tip as $(X=0, Y=0)$. Here, the strategy of [33] is closely followed. 
Expanding the $x$ and $y$ coordinates in powers of $R$, we then derive for the crack neighborhood (see Fig. 2.1):

$$
\left\{\begin{array}{c}
x=\alpha_{2} R \sin ^{2} \frac{\Theta}{2}+R^{3 / 2} F_{1}(\Theta)+R^{2} F_{2}(\Theta)+O(R) \ldots \\
y=\beta_{1} R^{1 / 2} \sin \frac{\Theta}{2}+R^{3 / 2} G_{1}(\Theta)+R^{2} G_{2}(\Theta)+O\left(R^{2}\right) \ldots
\end{array}\right\}
$$

where all unknown angular functions of $\Theta$ are found order by order, by solving the Euler-Lagrange equations, Eq. 6), and applying the incompressibility constraint:

$$
\left\{\begin{array}{ccc}
F_{1}(\Theta) & = & \frac{4}{3 \beta_{1}} \cos \frac{\Theta}{2}\left(1+2 \sin ^{2} \frac{\Theta}{2}\right) \\
F_{2}(\Theta) & = & \frac{1}{\beta_{1}^{2}} \sin ^{2} \frac{\Theta}{2}\left(b_{2}+a_{2} \sin ^{2} \frac{\Theta}{2}\right) \\
a_{2} & = & \alpha_{2}\left(6 b_{3}-\alpha_{2}^{2}\right)
\end{array}\right\}
$$

Notice that the leading order of $x$, Eq. (14), being $R$, the coefficient in front is named $\alpha_{2}$ and not $\alpha_{1}$ which would be the chosen coefficient in front of $R^{1 / 2}$. In a similar way, we obtain the functions corresponding to the horizontal displacement:

$$
\left\{\begin{array}{l}
G_{1}(\Theta)=\left(\frac{b_{3}}{\beta_{1}} \sin 3 \frac{\Theta}{2}-\frac{\alpha_{2}^{2}}{2 \beta_{1}} \sin \frac{\Theta}{2}\right) \\
G_{2}(\Theta)=\frac{\alpha_{2}}{\beta_{1}^{2}}\left(2 \sin \Theta-b_{4} \sin 2 \Theta\right)
\end{array}\right\}
$$

while the pressure reads:

$$
p=\frac{2 \alpha_{2}}{\beta_{1}} R^{1 / 2} \cos \frac{\Theta}{2}+\frac{8}{\beta_{1}^{2}} R\left(1+p_{1} \sin \left(\frac{\Theta}{2}\right)^{2}\right)+P_{0}
$$

$P_{0}$ and $p_{1}$ are constants which depend on the boundary conditions, Eq. (8). First, let us consider that there is no surface effects on the crack.

\subsubsection{Free crack lips, without capillary and surface effects}

If no surface energy is included, then $P_{0}=0$. On the crack, we have:

$$
s_{12}=-\frac{2}{\beta_{1}}\left(1+2 p_{1}\right) \sqrt{R} ; \quad s_{r r}=-\frac{2 \alpha_{2} R}{\beta_{1}^{2}}\left(b_{4}-4 p_{1}-3\right)
$$

which gives $p_{1}=-1 / 2$ and $b_{4}=1$.

\subsubsection{With surface effects}

Adding surface tension, the shear stress becomes

$s_{12}=-\frac{2 \alpha_{2} d_{0}+\beta_{1}^{2} P_{0}}{2 \beta_{1} \sqrt{R}}-\frac{2 \sqrt{R}}{\beta_{1}}\left(1+2 p_{1}-\frac{3 d_{0}}{\beta_{1}^{2}}\left(\alpha_{2}^{3}-b_{2}-8 \alpha_{2} b_{3}\right)\right)$

giving the change in the pressure:

$$
P_{0}=-2 \frac{\alpha_{2} d_{0}}{\beta_{1}^{2}} ; \quad p_{1}=-\frac{1}{2}+\frac{3 d_{0}}{2 \beta_{1}^{2}}\left(\alpha_{2}^{3}-b_{2}-8 \alpha_{2} b_{3}\right)
$$

Using the value obtained for $P_{0}$ from the cancellation of the shear stress, the leading term for the normal stress $s_{22}$ is a constant when the surface elastic energy is added: $s_{22}=2\left(\alpha_{2}^{3}-b_{2}-\right.$ $\left.6 \alpha_{2} b_{3}\right) d_{\mu} / \beta_{1}^{2}$. Consequently, cancellation of $s_{22}$ gives $b_{2}=-a_{2}$, according to Eq. 15 . Accordingly, we deduce that the elastic surface effect selects a coefficient entering the determination of $F_{2}(\Theta)$ which becomes $-a_{2} /\left(4 \beta_{1}^{2}\right) \sin (\Theta)^{2}$. The next order is not affected and $b_{4}$ keeps the same value, $b_{4}=-1$.

To conclude capillarity modified the pressure by adding a correction proportional to the parameter $d_{0}$ while the surface elastic stress selects the shape of the crack profile by fixing one of the angular function.
3.2. Local analysis near the singularity for the poro-elastic model

The Euler-Lagrange equations lead the following asymptotic expansion:

$$
\left\{\begin{array}{l}
x=\alpha_{1} R^{1 / 2} \cos \frac{\Theta}{2}+\alpha_{2} R \cos \Theta+R^{3 / 2} H_{1}(\Theta)+O\left(R^{2}\right) \\
y=\beta_{1} R^{1 / 2} \sin \frac{\Theta}{2}+\beta_{2} R \sin \Theta+R^{3 / 2} H_{2}(\Theta)+O\left(R^{2}\right),
\end{array}\right\}
$$

where

$$
\left\{\begin{array}{l}
H_{1}(\Theta)=\frac{1}{\alpha_{1}} \cos \frac{\Theta}{2}+\alpha_{3} \cos \frac{3 \Theta}{2} \\
H_{2}(\Theta)=\frac{1}{\beta_{1}} \cos \frac{\Theta}{2}+\beta_{3} \cos \frac{3 \Theta}{2}
\end{array}\right\}
$$

Let us examine now the boundary conditions.

\subsubsection{No surface effects}

Boundary conditions, Eqs. (8), when applied on the crack surface, become:

$$
\begin{gathered}
s_{12}=\frac{\alpha_{1}-\beta_{1} \Pi}{2 \sqrt{R}}-\frac{3 \sqrt{R}}{2 \alpha_{1} \beta_{1}}\left\{\beta_{1}\left(1+\alpha_{1} \alpha_{3}\right)+\Pi \alpha_{1}\left(1-\beta_{1} \beta_{3}\right)\right\} \\
s_{22}=\beta_{2}-\alpha_{2} \Pi+O(R)
\end{gathered}
$$

It is then possible to calculate the coefficients order by order: $\beta_{1}=\alpha_{1} / \Pi, \beta_{2}=\alpha_{2} \Pi$....suggesting that if the first line of coefficients is known, then all coefficients are fixed.

\subsubsection{Interfacial contributions on the surface crack}

Let us consider the effect of surface tension and surface elastic energy acting on the crack faces. It can be a way to select unknowns. Since the equivalent pressure is $1 / J_{2 D}$ in this model, we can assume that the surface pressure is $1 / x X$ and the normal nominal stress $s_{22}$ is modified

$s_{22}=\beta_{2}-\alpha_{2} \Pi+2 d_{0} \frac{\alpha_{2}^{2}}{\beta_{1}^{2}}+d_{\mu} \frac{2\left(1+\alpha_{2}^{2}\right)}{3 \alpha_{1}^{2} \alpha_{2}^{2} \beta_{1}}\left(\beta_{1}\left(\alpha_{2}-3 \alpha_{1}^{2} \alpha_{4}\right)+5 \alpha_{1} \beta_{2}\right)$,

while for the shear on the crack, it reads:

$$
s_{12}=\frac{\alpha_{1}-\beta_{1} \Pi+2 \alpha_{2} d_{0} / \beta_{1}}{2 \sqrt{R}}
$$

So the capillarity modifies the relationship between $\beta_{1}$, being now a function of $\alpha_{1}$ and $\alpha_{2}$, the stress surface energy gives a relation for $\beta_{2}$ as a function of the previous coefficients: $\alpha_{1}, \beta_{1}$ and $\beta_{2}$ but also $\alpha_{4}$. If $d_{\mu}=0$ but $d_{0} \neq 0$, then we get:

$\alpha_{2}=-\beta_{1} \mathcal{A} ; \beta_{2}=-\mathcal{A}\left(2 \mathcal{A} d_{0}+\beta_{1} \Pi\right)$ with $\mathcal{A}=\left(\alpha_{1}-\beta_{1} \Pi\right) /\left(2 d_{0}\right)$

\subsection{Local analysis for the plane stress model}

The analysis is more delicate for this model since it tremendously depends on the power of the expansions in $x$ and $y$, so on initial guess. Assuming as an example that $x \sim R^{a}$ and $y \sim R^{1 / 2}$, we easily obtain that $J_{2 D} \sim R^{-3 / 2+a}$ and the apparent pressure $p \sim R^{3(3 / 2-a)}$. Also the left-hand side of the Euler-Equation, Eq. (6), is of order $R^{(a-2)}$ if the first terms are not equal to the Real Part of an holomorphic function. The right-hand-side is of order $R^{3(3 / 2-a)} R^{1 / 2} R^{-2}$ which gives $R^{3(1-a)}$. The same analysis can be achieved for the second Euler-Lagrange equation and 
the right-hand side in this case behaves as $R^{3(3 / 2-a)} R^{a} R^{-2}$ so as $R^{5 / 2-2 a}$. Let us choose $a=1$ as in Section 3.1 and $a=1 / 2$ as for the poro-elastic model (Section 3.2). If $a=1$, automatically we derive the following expansion for $x$ and $y$ which respects both the scaling estimate made above and the symmetry:

$$
\left\{\begin{array}{r}
x=\Sigma_{2,3}(R, \Theta)+R^{2} K_{1}(\Theta)+O\left(R^{2}\right) \\
y=\tilde{\Sigma}_{1,4}(R, \Theta)+R^{5 / 2} K_{2}(\Theta)+O\left(R^{5 / 2}\right)
\end{array}\right.
$$

If $a=1 / 2$, then we derive:

$$
\left\{\begin{array}{l}
x=\Sigma_{1,6}(R, \Theta)+R^{7 / 2} K_{3}(\Theta)+O\left(R^{7 / 2}\right) \\
y=\tilde{\Sigma}_{1,6}(R, \Theta)+R^{7 / 2} K_{4}(\Theta)+O\left(R^{7 / 2}\right)
\end{array}\right.
$$

with

$$
\left\{\begin{array}{l}
\Sigma_{j, k}(R, \Theta)=\sum_{j}^{k} \alpha_{j} R^{j / 2} \cos j \Theta / 2 \\
\tilde{\Sigma}_{j, k}(R, \Theta)=\sum_{j}^{k} \beta_{j} R^{j / 2} \sin j \Theta / 2
\end{array}\right.
$$

Concerning the choice of the first terms in Eq. 26, one can also choose $x \sim R^{1 / 2}$ and $y \sim R$, but both choices lead to singularities on the crack lips for the functions $K(\theta)$ representing the first non holomorphic term in the expansion. So we eliminate such choice and select the expansion given by Eq. 27). Solving Eqs. (6) gives the followings results for $K_{3}$ and $K_{4}$ :

$$
\left\{\begin{array}{l}
K_{3}(\Theta)=\frac{8}{\alpha_{1}^{3} \beta_{1}^{2}} \cos \frac{\Theta}{2}+k_{3} \cos \frac{7 \Theta}{2} \\
K_{4}(\Theta)=\frac{8}{\alpha_{1}^{2} \beta_{1}^{3}} \cos \frac{\Theta}{2}+k_{4} \sin \frac{7 \Theta}{2}
\end{array}\right.
$$

Boundary conditions Eqs. (8) on the crack surface are transformed into:

$$
s_{12}=\frac{\alpha_{1}}{2 \sqrt{R}}-\frac{3}{2} \alpha_{3} \sqrt{R} ; \quad s_{22}=-64 \alpha_{2}\left(\frac{R}{\alpha_{1} \beta_{1}}\right)^{3}
$$

The last relation being valid if $\beta_{2}=\beta_{4}=\beta_{6}=0$. The only way to cancel the shear stress at the tip is to choose $\alpha_{1}=0$ or to introduce surface effect. However taking $\alpha_{1}=0$ means to choose Eq. 26 that we must reject because of singularities of the angular function $K_{1}$.

\subsubsection{Capillarity or surface stress}

The previous expansion fails in absence of surface effects that ${ }^{200}$ we introduce now, following Eq. (8). Defining the parameter and after revisiting the local expansion allows to eliminate the shear stress singularity giving:

$$
s_{12}=\left(\frac{\alpha_{1}}{2}+d_{0} \frac{\alpha_{2}}{\beta_{1}}\right) \frac{1}{\sqrt{R}}+O(\sqrt{R})
$$

So $\alpha_{2}=-\alpha_{1} \beta_{1} /\left(2 d_{0}\right)$ and the normal stress component is then

$$
s_{22}=\beta_{2}+\alpha_{1}^{2} /\left(2 d_{0}\right)-2 d_{\mu}\left(1+2\left(3 d_{0} / \alpha_{1} \beta_{1}\right)^{4}\right)+O(R)
$$

In this case, capillarity is necessary to allow the existence of the crack by cancelling the singularity of the shear stress and avoiding the choice Eq. 26). The calculation can be achieved order by order, in particular $\beta_{2}$ does not vanish and is equal to $-\alpha_{1}^{2} /\left(2 d_{0}\right)$, if the elastic surface stress is not considered. Here again, it simply modifies the values of coefficients. However, one may notice that 2 coefficients remain free at this stage $\alpha_{1}$ and $\beta_{1}$. We conclude that for plane stress, it is possible to find a correct local expansion around the crack tip if capillarity is included.

\subsection{Partial conclusion on the local analysis}

We have found a way to calculate order by order, the crack shape in finite elasticity. Such asymptotic analysis seems systematic but its validity is restricted to the convergence of the series expansions, difficult to prove for series of 2 variables. As shown by the 3 cases investigated here, $\beta_{1}$ remains an unknown coefficient while $\alpha_{1}$ vanishes or is a function of $\beta_{1}$ or remains a free degree of freedom. Concerning the crack shape derived by imposing $\Theta= \pm \pi$, in the 3 cases, it is parabolic and since the crack is in tension, we anticipate the fact that $\beta_{1}<0$. In the next paragraph, we use the technique of path-independent integral to relate $\beta_{1}$ to the tension induced by drying or resorption.

\section{Evaluation of unknowns via path-independent integrals}

Path independent integrals are a very useful tool to fix constants in the asymptotics around singularities. It has been considered a lot in crack problems [23] mostly in the context of linear elasticity. However, it has been extended to nonlinear elasticity in various configurations [27] and when elasticity is combined to other singular fields such as electrostatic [25, 26]. For a review of the foundation of this technique and extension to other integrals in other contexts, see [24]. Here, we use the path independent $J$ integral (in the reference configuration) valid for finite elasticity. $J$ being a vector, we focus on $J_{1}$ :

$$
J_{1}=\oint\left(W N_{1}-N_{k} s_{i k} F_{i 1}\right) d l=0
$$

This integral cancels for a closed contour when it does not enclose a singularity. So we choose the contour which follows the slab of lateral dimension $\Lambda$ in the reference configuration, or follows the unit of a periodic network of cells in the same geometry. Our contour contains the upper border, includes the two faces of the crack and avoids the neighborhood of the crack tip (the circle in Fig.(2.1), then the 2 lateral sides of the cell and finally an horizontal path far from the tip. For reason of symmetry, contribution to $J_{1}$ cancels on the crack lips and on contour lines parallel to the $X$ axis (exterior vertical border of the slab). Indeed $N_{1}=0$ in this case and assuming that the stress and stretch, far away from the crack, remain diagonal, the second term in Eq. (33) vanishes.

\subsubsection{The J-integral around the crack tip}

Focussing on the singularity, it is easy to show (and it is known) that a crack tip in $R^{1 / 2}$ contributes to the $J$ integral by a constant which can be evaluated by considering a small circular contour around it, with a radius $R=\epsilon, \epsilon \rightarrow 0$ (see the contour in 
Fig.(2.1). In the 3 models, the energy term, being independent of $\Theta$, cancels when integrated along the small circle and only the stress contribution (second term in Eq. (33)) contributes:

$$
\begin{aligned}
J_{\text {crack }} & =-R \int_{-\pi}^{\pi} d \Theta \cos \Theta\left(s_{11} x_{X}+s_{21} y_{X}\right) \\
- & R \int_{-\pi}^{\pi} d \Theta \sin \Theta\left(s_{12} x_{X}+s_{22} y_{X}\right)
\end{aligned}
$$

in the limit $R \rightarrow 0$. In the case of volumetric growth, we have $\alpha_{1}=0$ and the pressure in the stress gives a subdominant contribution. Only $s_{22} \sim \beta_{1} \cos \Theta / 2 /(2 \sqrt{R})$ and $s_{21}=$ $\left.-\beta_{1} \sin \Theta / 2\right] /(2 \sqrt{R})$ contribute finally leading to:

$$
J_{\text {crack }}=\frac{\beta_{1}^{2} \pi}{4}
$$

For the poro-elastic model and the plane-stress model with capillarity, again the "fictive" pressure gives no contribution around the crack and

$$
J_{\text {crack }}=\frac{\left(\beta_{1}^{2}-\alpha_{1}^{2}\right) \pi}{4}
$$

Since, for the poro-elastic model, $\alpha_{1}=\Pi \beta_{1}$ in absence of surface tension, we also get: $J_{\text {crack }}^{\text {poro }}=\beta_{1}^{2}\left(1-\Pi^{2}\right) \pi / 4$. If surface $_{220}$ tension exits but remains very small, a good estimation would be $\alpha_{1} \sim 0$, in plane stress. In this case, surface tension plays the role of a singular perturbation.

\subsubsection{The J-integral in the volumetric or plane stress model of resorption}

We now need to close the contour but only ideal situations give exact results, even in linear elasticity. Very often, in our geometry, the choice is made to consider a crack of finite length with 2 tips, then to consider the horizontal paths at both infinities, where it is possible to play with eventually 2 different experimental conditions. Here we imagine that the cell is large enough so that the crack does not perturb too much the imposed ${ }^{230}$ stress field due to the volumetric process. In plane strain, one easily gets for the elastic stretch tensor: $\mathbf{F}_{\mathbf{e}}=\operatorname{Diag}\left(g_{2}, 1 / g_{2}, 1\right)$. The second term in Eq. (33) vanishes on both horizontal paths parallel to the $Y$ axis while the lateral borders of the strip are under tension with $s_{22}=1 / g_{2}-g_{2}^{3}$, if $g_{2}<1$. So only the eigenvalue $g_{2}$ is responsible for the tensile stress in this geometry. $\left.J_{\text {border }}=-1 / 2\left(g_{2}^{2}-1\right)^{2} / g_{2}^{2}\right)\left(g_{2} \Lambda\right)$ which gives for the coefficient $\beta_{1}$ :

$$
\beta_{1}=2 \frac{g_{2}^{2}-1}{g_{2}} \sqrt{\frac{g_{2} \Lambda}{\pi}}
$$

The choice is made of a negative sign for $\beta_{1}$ which is physically more relevant. In plane stress elasticity, one needs to take into account the stretch in the third dimension in the energy estimate. Assuming the same base state as before with $y=\tilde{Y}$ so $\partial y / \partial Y=1 / g_{2}$, we deduce from Eq. (7): $\partial x / \partial X=\sqrt{g_{2}}=\lambda_{3}$ which changes the value of the energy density and finally:

$$
\beta_{1}=-2 \sqrt{2 \sqrt{g_{2}}+1 / g_{2}^{2}-3} \sqrt{\frac{g_{2} \Lambda}{\pi}}
$$

when $\alpha_{1}$ is negligible.

\subsubsection{The J-integral and the poro-elastic model}

We assume the unit cell in three dimensions. Drying is an isotropic process and all dimensions are characterized by a drying coefficient at time $t$. We call $\bar{v}$ the volume decrease per unit volume, then $\bar{v}$ corresponding to $g_{1} g_{2} g_{3}$. Here again, the axial direction imposed by the geometry gives $\mathbf{F}=\operatorname{Diag}(\bar{v}, 1,1)$ (other experimental choices can be made, like in [11]). Evaluation of the stress $s_{11}$ at the free upper border leads to $\Pi=\bar{v}-1 / \bar{v}$, which proves that in drying, the chemical potential is negative. One can calculate now the $J$ integral on the horizontal paths, we get on both lines the same value and using $J_{\text {crack }}^{\text {poro }}$, it reads:

$$
\beta_{1}^{2}\left(1-\Pi^{2}\right) \pi / 4=\bar{v}^{2}-1-2 \log \bar{v}-2 \Pi ; \Pi=\bar{v}-\frac{1}{\bar{v}}
$$

which finally gives

$$
\beta_{1}=-2 \sqrt{\frac{(\bar{v}-2) \Pi-2 \log \bar{v} / \bar{v}^{2}}{1-\Pi^{2}}} \sqrt{\frac{\bar{v}^{1 / 3} \Lambda}{\pi}}
$$

Notice that the coefficient $\beta_{1}$, calculated in Eqs. 36 37 39, corresponds to the rescaled coordinates for $(X, Y, Z)$. The corresponding $\tilde{\beta}_{1}$ in the coordinate system $(\tilde{X}, \tilde{Y}, \tilde{Z})$ will be $\tilde{\beta}_{1}=$ $\beta_{1} / \sqrt{g_{2}}$.

\section{Conclusion}

We have presented a local analysis of cracks in finite elasticity in two space dimensions. Three models are considered based on the the Neo-Hookean elasticity. The method is straightforward and the J-integral allows to relate the main coefficients near the tip to the resorption or drying rates at long distances, then showing the consistency of the analysis. For completeness, surface tension and surface elastic energy are added, although these effects do not change really the results, appearing mostly as perturbation, except for the plane stress model. It is known that drying induces cracks (see Fig.(1)), however when growth is anisotropic, only the growth strain perpendicular to the crack $g_{2}$ is responsible for the crack formation. Although the theory of cracks is rather well established, little is known in the context of finite elasticity and in particular when the tissue itself is anisotropic. However, experimentalists in biophysical laboratories make laser cuts on embryos to access the residual stresses due to growth. This technique has the advantage to obtain the growth pre-stress or pre-strain in different parts of the embryo without killing it. There is no doubt that more works are required on fracture in both anisotropic growth and anisotropic tissues [17].

\section{Acknowledgments}

This work is dedicated to the memory of Gérard Maugin, who has had a major influence on the mechanics of solids in France and abroad. I thank him for his leniency with regard to my modest contributions in the field of biomechanics. I also thank Ivan C. Christov, Michel Destrade, and Anthony Rosato for initiating and organizing this special issue. It is 
so a pleasure for me to acknowledge the CISM-IUTAM International Summer School 2017 (Udine, Italy) "Growth ${ }^{320}$ and Remodeling in Soft Biological tissue" organized by J. Humphrey and C. Cyron, as it gave me the opportunity to present this work in the context of singularities in soft tissue elasticity.

[1] A.E. Lobkovsky, Boundary layer analysis of the ridge singularity in a thin plate, Phys. Rev. E 53 (4) (1996) 3750

[2] M. Ben Amar, Y. Pomeau Crumpled paper, Proc.Roy. Soc. Lond. A $453^{330}$ (1997) 729-755.

[3] V. Brunck, F. Lechenault, A. Reid, M. Adda-Bedia, Phys. Rev. E 93 (2016) 033005

[4] M. Ben Amar, M Wu, Patterns in biofilms: from contour undulations to fold focussing, Eur. Phys. Lett 108 (2014) 38003-08

[5] L. Landau, E. Lifchitz, E.Theorie de lélasticité. Moscow: Mir (1990)

[6] A. Gray, Modern differential geometry of curves and surfaces, Boca Raton: FL: Chemical Rubber Company (1993)

[7] T. Tanaka it et al. Mechanical instability of gels at the phase-transition, Nature 325 (1987) 796-798.

[8] E. Matsuo, T. Tanaka, Patterns in shrinking gels, Nature 358 (1992) 482485.

[9] V. Trujillo, J. Kim, R.C. Hayward, Creasing instability of surface attached hydrogels, Soft Matter 4 (2008) 564569.

[10] W. Hong, X. Zhao, J. Zhou, Z. Suo, A theory of coupled diffusion and large deformation in polymeric gels, J. Mech. Phys. Solids 56 (2008) 1779-1793.

[11] J. Dervaux, M. Ben Amar, Buckling condensation in constrained growth, JMPS (2011) 538-560.

[12] B. Li, F. Jia, Y.P. Cao, X.Q. Feng, H. Gao, Surface wrinkling patterns on a core-shell soft sphere. Phys. Rev. Lett. 106 (2011) 234301. (doi:10.1103/PhysRevLett. 106.234301)

[13] F. Jia, B. Li, Y.P. Cao, W.H. Xie, X.Q. Fen, Wrinkling pattern evolution of cylindrical biological tissues with differential growth Phys. Rev. E 91 (2015) 012403. (doi:10.1103/PhysRevE.91.012403)

[14] M. F. Benedetto, S. Berrone, S. Pieraccini, S. Scialo, The virtual element method for discrete fracture network simulations, Journal of Computational Physics, 280, 135-156 (2014)

[15] Y Couder, L Pauchard, C Allain, M Adda-Bedia \& S. Douady, The leaf venation as formed in a tensorial field. Eur. Phys. J. B 28, 135-138 (2002)

[16] C. Allain \& L. Limat, Regular patterns of cracks formed by directional drying of a collodial suspension.Phys. Rev. Lett. 74, 2981-2984 (1995)

[17] T. Kim Vuong-Brender, M. Ben Amar, J. Pontabry, M. Labouess,e The interplay of stiffness and force anisotropies drive embryo elongation eLife 6 :e23866 (2017)DOI: 10.7554/eLife.23866

[18] D. Bonn, H. Kellay, M. Prochnow, K. Ben-Djemiaa, J. Meunier, Delayed fracture of an inhomogeneous soft solid. Sciences 280 (1998) 265-267.

[19] M. Adda-Bedia, M. Ben Amar Stability of quasi-equilibrium cracks under uniaxial loading, Phys. Rev. Lett. 76(9) (1996) 1497-1500.

[20] M. Adda-Bedia, R. Arias, M. Ben Amar , F. Lund Dynamic instability of brittle fracture, Phys. Rev. Lett. 82 (1999) 2314-2317.

[21] E. Yoffe, The moving Griffith crack, Phil. Mag. 42 (330) (1951) 739-750.

[22] G. Freund, Dynamic fracture mechanics, Cambridge University Press (1990)

[23] J.R. Rice, A path independent integral and the approximate analysis of strain concentration by notches and cracks. J. Appl. Mech.35 (1968) 379386 .

[24] J.K. Knowles and E. Sternberg, On a class of Conservation Laws in Linearized and Finite Elastostatics, Archive for rational mechanics and analysis 1972 Springer.

[25] G.A. Maugin, M. Epstein,The Electroelastic Energy-Momentum Tensor. Proc. Roy. Soc. A 433 (1991) 299-312.

[26] C. Dascalu, G.A. .Maugin, Energy-release rates and path-independent integrals in electroelastic crack propagation, International Journal of Engineering Science 32,(1994) 755-765.

[27] C. Trimarco, G.A. Maugin, Bui's path-independent integral in finite elasticity Meccanica 30 (1995) 139-145.

[28] A.K. Rodriguez, A. Hoger, A. McCulloch, Stress-dependent finite growth in soft elastic tissue. J. Biomech. 27 (1994) 455-467.
[29] A. Goriely, The Mathematics and Mechanics of Biological Growth.Interdisciplinary Applied Mathematics, Springer (2017)

[30] C.Y. Hui, A. Jagota, Surface tension, surface energy, and chemical potential due to their difference 29 (36) (2013) 11310-11316.

[31] R.W. Style, A. Jagota, C.Y. Hui, E. R. Defresne. Elastocapillarity: Surface Tension and the Mechanics of Soft Solids. Annual Review of Condensed Matter Physics 8 (2017) 99-118.

[32] M. Ben Amar, P. Ciarletta, Swelling instability of surface-attached gels as a model of tissue growth under geometric constraints. JMPS 58 (2010) 935-954.

[33] R.A. Stephenson, The equilibrium field near the tip of a crack for finite plane strain of incompressible elastic materials Journ. Elast. 12(1) (1992) 65-98.

[34] JK Knowles, E Sternberg An asymptotic finite-deformation analysis of the elastostatic field near the tip of a crack.Journal of Elasticity 3 (2) (1973) 67-107. doi:10.1007/BF00045816. 